\title{
Analysis of Paint Flow Pulsations during High-Speed Rotary Bell Atomization
}

\author{
Walter Oswald ${ }^{1, *} \mathbb{D}$, Jan Lauk ${ }^{1}$, Lutz Gödeke ${ }^{2}$, Peter Ehrhard ${ }^{2}$ and Norbert Willenbacher ${ }^{1}$ \\ 1 Applied Mechanics, Institute of Mechanical Process Engineering and Mechanics, KIT, Gotthard-Franz-Straße 3, \\ 76131 Karlsruhe, Germany; uodru@student.kit.edu (J.L.); norbert.willenbacher@kit.edu (N.W.) \\ 2 Fluid Mechanics, Biochemical and Chemical Engineering, TU Dortmund, Emil-Figge-Straße 68, \\ 44227 Dortmund, Germany; lutz.goedeke@tu-dortmund.de (L.G.); peter.ehrhard@tu-dortmund.de (P.E.) \\ * Correspondence: walter.oswald@kit.edu; Tel.: +49-721-608-42805
}

Received: 23 September 2019; Accepted: 13 October 2019; Published: 17 October 2019

\begin{abstract}
High-speed rotary bell atomization is the preeminent coating technique in the automotive industry. It is widely accepted that a narrow droplet size distribution and constant spray are necessary in order to guarantee uniform film thickness and high-quality appearance. This may be deteriorated by paint flow pulsations. So far, however, no studies exist regarding such fluctuations quantitatively for this type of atomizers. We fill this gap using image analysis of high-speed recordings close to the bell edge. We could show that the fundamental pulsation frequency increases linearly with rotational speed. A ratio of pulsation frequency and true rotational speed of about 3 was found, indicating that pulsations were initiated mainly by the three struts of the distributor disc. The coefficient of variation, i.e., the amplitude of fluctuation increased with decreasing liquid volume rate and rotational speed. Beyond that, we could show that the formation of droplets larger than $100 \mu \mathrm{m}$, which are assumed to cause paint defects, is promoted by the degree of fluctuation. These findings may stimulate development of bell cups showing less paint flow pulsations.
\end{abstract}

Keywords: pulsation; high-speed rotary bell atomization; automotive coatings; image analysis; Fourier transformation

\section{Introduction}

Automotive coatings are usually applied via high-speed rotary bell atomization. This coating technique provides the desired high-quality appearance and a high transfer efficiency at short process times. During this operation process, the paint is supplied to a distributor disc which is connected to the rotary bell. A thin film of the paint is formed and flows to the bell edge due to centrifugal force. Finally, atomization occurs by direct drop formation, ligament formation, or sheet formation depending on material properties such as density $\rho$, surface tension $\sigma$, and shear viscosity $\eta_{\mathrm{s}}$ of the liquid and on application parameters including rotational speed $n$ and the volume flow rate $\dot{V}[1,2]$. Ogasawara et al. [3] investigated the breakup mechanism for serrated bell cups and showed that the presence of serrations supports droplet formation caused by the disintegration of ligaments. During high-speed rotary bell atomization, a shaping air flow is used in order to deflect the radially accelerated spray and to obtain an axial flow of the paint towards the substrate.

One of the major demands in this process is the assurance of a uniform film thickness of the final coating. This can be affected detrimentally by an unsteady flow of the paint. Partially, this may be compensated by adjusting the travel path of the spray head. Nevertheless, it is essential to describe paint flow pulsations in order to avoid corresponding deficiencies in coating quality. 
In the past, various experimental techniques were used to characterize pulsations during atomization quantitatively. Kourmatzis et al. 2016 [4] analyzed the velocity time series obtained from Laser Doppler anemometry using fast Fourier transformation in order to examine the instability frequency for a combined effervescent and airblast atomizer. Ariyapadi et al. 2001 [5] determined the frequency of artificially generated pulsations by the measurement of the time interval between consecutive signals of a phase Doppler particle analyzer. Schlinge et al. 2013 [6] and Schröder et al. 2013 [7] determined the time-dependent volume droplet diameter using laser diffraction spectroscopy and calculated the fundamental frequency fitting a sine function to the data measured for effervescent atomization. Moreover, photographic methods have also been used to investigate pulsations. Mullis et al. 2013 [8] analyzed close-coupled gas atomization and detected paint flow fluctuations by plotting the temporal change of the mean grey value of images recorded during this process. Schlinge et al. 2013 [6] plotted the fraction of pixels defined as spray versus corresponding time and determined the pulsation frequency manually. By contrast, Ting et al. 2005 [9] obtained a frequency spectrum applying a discrete Fourier transformation to the dataset of images yielding a time-dependent number of pixels assigned to the spray. The degree of fluctuation itself was defined by Kourmatzis et al. 2016 [4] as the standard deviation of the recorded spray area.

It may be also important to know how pulsations affect spray characteristics. Schröder et al. 2013 [7] found that increasing pulsation frequency decreased the Sauter mean diameter (SMD) and corresponding standard deviation. By contrast, Im et al. 2009 [10] and Kang et al. 2016 [11] showed that self-pulsation of a gas-liquid swirl coaxial injector led to an increase of the SMD.

Soma et al. 2015 [12] numerically studied the effect of pulsations originating from a gear pump on the flow pattern of a high-speed rotary bell atomizer. They could show that the frequency of flow rate pulsations in the liquid supply nozzle is the same as that of the fluctuations of film thickness at the bell edge. However, the amplitude of film thickness variation in the bell cup was only $30 \%$ of the amplitude of flow rate fluctuation in the nozzle. Furthermore, Salazar [13] investigated unsteady flow of paint on rotary bells cups using computational modeling. He demonstrated that droplet formation is a pulsating-like phenomenon, since vortex waves are created on the bell surface. On the one hand, serrations are overflowed, leading to sheet formation; on the other hand, ligaments with small diameters are formed at the bell edge. As a consequence, a broad droplet size distribution is expected.

Here, we present the first experimental study on pulsations occurring during high-speed rotary bell atomization. The paper is organized as follows: First, we present the image processing and analyzing tools for the determination of the frequency and the degree of fluctuation. Next, we discuss the effect of application parameters (volumetric flow rate and rotational speed) on these pulsation characteristics and examine the source of pulsations. Finally, we correlate the degree of fluctuation with the occurrence of big droplets in the spray which are assumed to deteriorate the coating quality.

\section{Materials}

An industrial waterborne basecoat (BASF Coatings $\mathrm{GmbH}$, Münster, Germany) with a surface tension of $\sigma=27 \mathrm{mN} / \mathrm{m}$ and a density of $\rho=1040 \mathrm{~kg} / \mathrm{m}^{3}$ was used. This basecoat exhibits strong shear thinning behavior (Figure 1). Shear rate at the bell edge is approximated to vary between $2 \times 10^{4}$ and $6 \times 10^{5} \mathrm{~s}^{-1}$ [14], depending on volumetric flow rate and rotational speed. In this range, the sample has a shear viscosity of $\eta_{\mathrm{s}}=0.01-0.04 \mathrm{~Pa} \cdot \mathrm{s}$. 


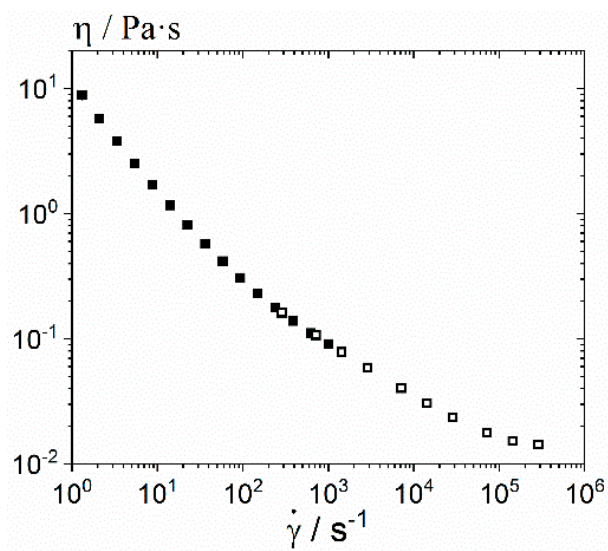

Figure 1. Shear viscosity $\eta_{\mathrm{s}}$ as a function of shear rate $\dot{\gamma}$ for the automotive basecoat. Closed symbols refer to data from rotational rheometry (coaxial cylinder system DIN-Z20), open symbols correspond to capillary rheometry (radius $r_{\text {cap }}=0.25 \mathrm{~mm}$ and length $l_{\text {cap }}=40 \mathrm{~mm}$ of the capillary) data.

\section{Experimental Methods}

\subsection{Atomization}

Experiments were performed in an air-conditioned spray cabin with constant temperature of $25 \pm 2{ }^{\circ} \mathrm{C}$ and relative humidity of $\varphi_{\text {air }}=65 \%$. A filtered vertical air stream with a velocity of $0.3 \mathrm{~m} / \mathrm{s}$ ensured a dust-free environment. We have atomized the basecoat using a high-speed rotary bell atomizer EcoBell2 (Dürr AG, Stuttgart, Germany). The bell cup (Figure 2) had a contour angle of $\alpha=62^{\circ}$ and a maximal radius of $r_{\text {bell }}=65 \mathrm{~mm}$ including serrations at the bell edge with a width of $0.12 \mathrm{~mm}$. The distributor disc had a radius of $r_{\text {disc }}=11 \mathrm{~mm}$ and was connected to the bell edge by three struts. Nominal rotational speed and volumetric flow rate were varied in the ranges $n=10-50 \mathrm{krpm}$ and $\dot{V}=200-550 \mathrm{~mL} / \mathrm{min}$, respectively. The shaping air flow was kept constant at a value of $340 \mathrm{Nl} / \mathrm{min}$.

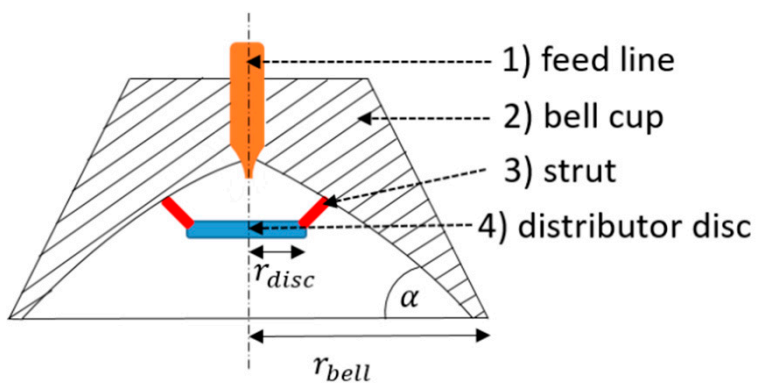

Figure 2. Schematic image of the rotary bell cup.

\subsection{Pulsations}

For the determination of pulsations, 3000 images were recorded directly below the bell edge at a resolution of $256 \times 520$ pixels and a frame rate of $100,000 \mathrm{fps}$ using a high-speed camera Fastcam SA-Z (Photron, Tokyo, Japan) equipped with the telecentric lens S5LPJ1007/216 (Sill Optics, Wendelstein, Germany) showing a magnification of 1.0 and a working distance of $91 \mathrm{~mm}$. Object illumination was done using light source LED-P40 (SMETEC, Erkelenz, Germany). Next, these images were processed using a self-written MATLAB (version R2017b, MathWorks, Natick, MA, USA) code, as presented in Figure 3. First, brightness of the raw images (a) was balanced and the contrast between background and spray was improved (b). After that, images were binarized applying an adaptive threshold method (c). The bell cup was removed from the binary images $(\mathrm{d})$ and small objects like picture noise of the raw images or sharpening noise were eliminated (e). In the final step of image processing, images were inverted, i.e., the binary value of the pixels was switched (f). 


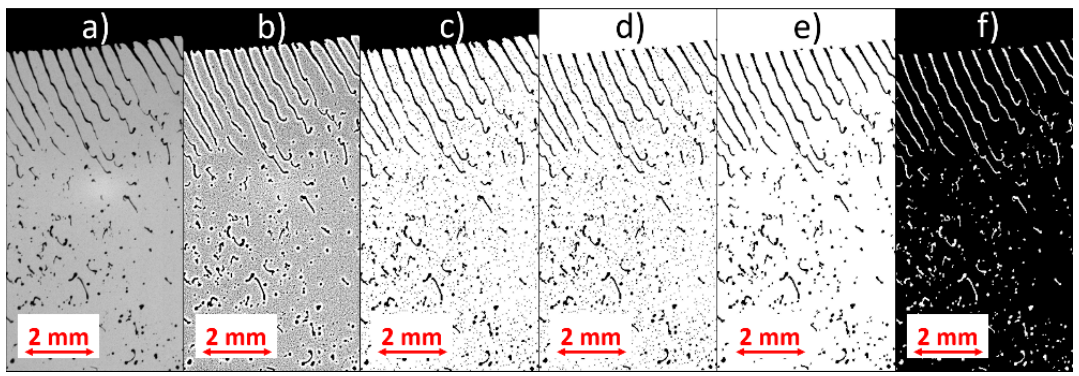

Figure 3. Image processing for the determination of pulsations by MATLAB: (a) raw image, (b) improvement of contrast, (c) binarization, (d) removal of bell edge, (e) suppression of noise, and (f) inversion.

For every processed image, the number of remaining white pixels was summed up and scaled with the area of the entirety of pixels. Plotting these values against the corresponding time already indicates the presence of pulsations as shown exemplarily in Figure 4 for the atomization of the basecoat at $\dot{V}=350 \mathrm{~mL} / \mathrm{min}$ and $n=10 \mathrm{krpm}$. These data were transformed via fast Fourier transformation into a frequency spectrum, as shown in Figure 5. We detected a fundamental frequency of $430 \mathrm{~Hz}$ and further multiples of this frequency. In some experiments, these multiples show higher normalized amplitude values than the fundamental frequency. Nevertheless, we focused on the first frequency with a value of normalized amplitude higher than 0.5 . The coefficient of variation $(\mathrm{CV})$ calculated from the spray area of all images was used in order to quantify the degree of fluctuation.

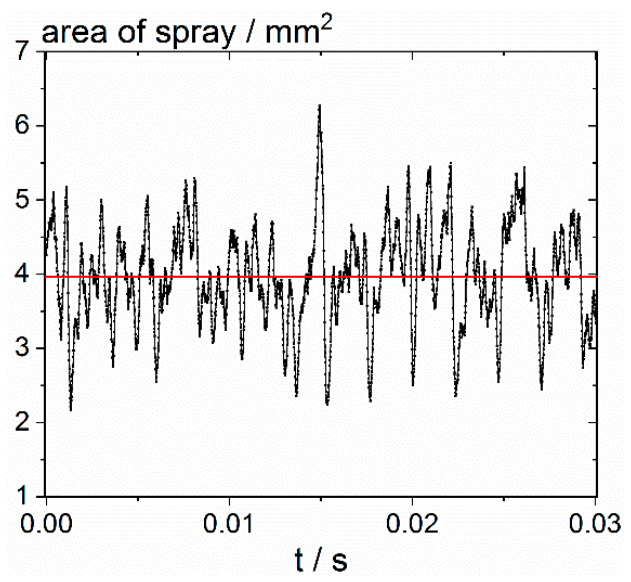

Figure 4. Time evolution of white pixel area during atomization at $n=10 \mathrm{krpm}$ and $\dot{V}=350 \mathrm{~mL} / \mathrm{min}$. Red line represents mean value.

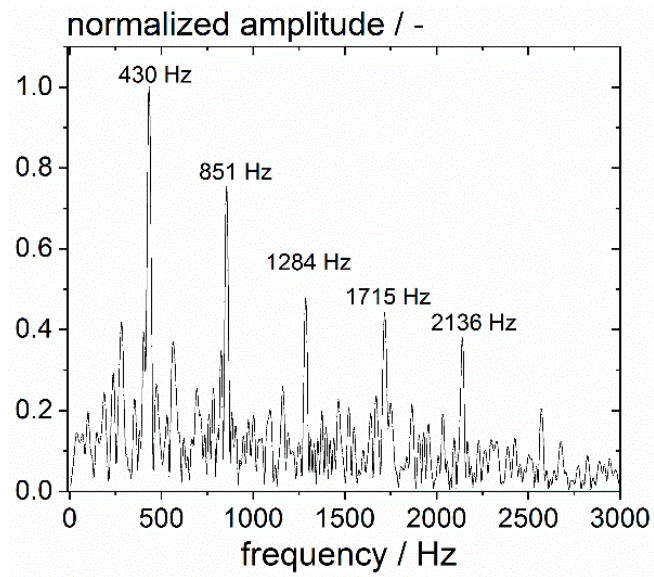

Figure 5. Frequency spectrum of data from Figure 4. Amplitude is normalized with the value of the highest amplitude of the spectrum. 
Droplet size measurements were performed using a Dantec FiberFlow ${ }^{\circledR}$ Phase Doppler anemometer (Dantec Dynamics GmbH, Ulm, Germany). This technique is based on laser Doppler anemometry, where droplets interfere with transmitting light inside a defined measurement volume, resulting in unique scattered light signals. These signals contain information about the Doppler frequency from which the velocity of the scattering objects-i.e., the droplets-can be calculated. This method was expanded by two additional receiving units, enabling measurements of the phase shift between the Doppler signals, thus enabling the calculation of droplet size and sphericity. All droplets showing a sphericity smaller 0.9 were discarded from further analysis. The initial measurement volume was located at a distance of $25 \mathrm{~mm}$ tangentially to the edge of the tilted $\left(45^{\circ}\right)$ bell cup. Droplets were measured at a range of positions in radial direction in order to characterize the entire spray cone.

\section{Results and Discussion}

Figure 6a shows the fundamental frequency from the fast Fourier transformation of paint flow pulsations as described above for different flow rates and rotational speeds. Frequency increases linearly with rotational speed irrespective of volumetric flow rate. However, increasing volumetric flow rate leads to a slight decrease of frequency, especially at high $n$. In Figure $6 \mathrm{~b}$, the ratio of fundamental frequency and nominal rotational speed is plotted as a function of nominal rotational speed $n$. This value is essentially independent of $n$, and lies between 2.4-2.8 depending on volumetric flow rate of the basecoat. Hence, for every rotation, approximately three pulsations occur. This is equivalent to the number of struts connecting the distributor disc to the bell cup. Hence, we conclude that fluctuations of the spray are mainly caused by these struts. The number of pulsations per nominal rotation is lower than 3 , and slightly decreases with increasing volumetric flow rate. This is because the true rotational speed $n_{\text {true }}$ is lower than $n$ and decreases with volumetric flow rate as shown exemplarily for $n=20 \mathrm{krpm}$ in Figure $6 \mathrm{c}$ and, accordingly, the ratio frequency $/ n_{\text {true }}$ is approximately 3 . True rotational speed $n_{\text {true }}$ was determined from high-speed recordings of the bell cup rotation.

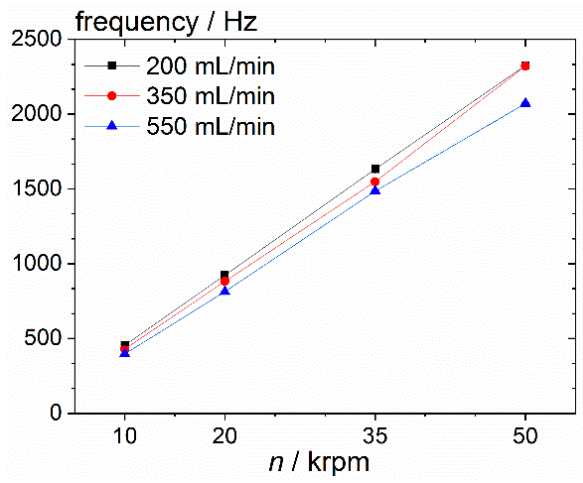

(a)

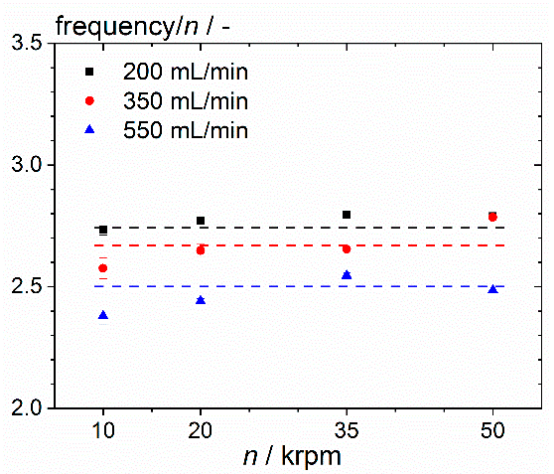

(b)

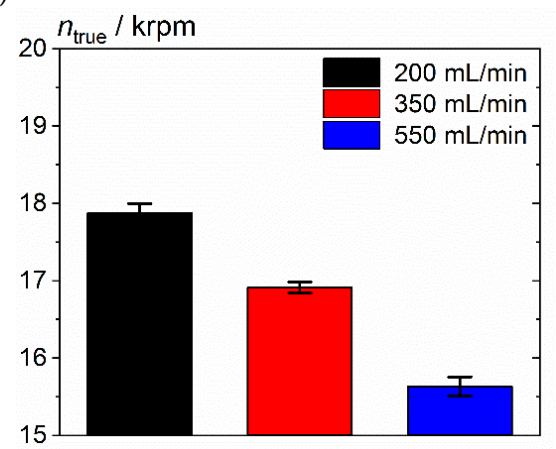

(c)

Figure 6. (a) Fundamental pulsation frequency vs. nominal rotational speed $n$ for different volumetric flow rates $\dot{V}$. (b) Pulsation frequency $/ n$ vs. nominal rotational speed $n$ for different volumetric flow rates $\dot{V}$. The lines are to guide the eye. (c) True rotational speed $n_{\text {true }}$ for different volumetric flow rates $\dot{V}$ during atomization at a nominal rotational speed of $n=20 \mathrm{krpm}$. 
The degree of fluctuation is characterized by the coefficient of variation calculated from the amount of spray for all 3000 images taken during a spray experiment. Figure 7 shows that this parameter decreases with increasing rotational speed. We assume that increasing rotational speed, i.e., increasing centrifugal force, promotes levelling of the film thickness at the bell edge. As a consequence, the amplitude of pulsations decreases and, hence, CV decreases as well. Furthermore, a slight decrease of $\mathrm{CV}$ with increasing volumetric flow rate is observed for nominal rotational speeds $n \geq 35 \mathrm{krpm}$. The centrifugal force acting on the paint increases with increasing volumetric flow rate as well and the relative amplitude and CV are expected to decrease accordingly.

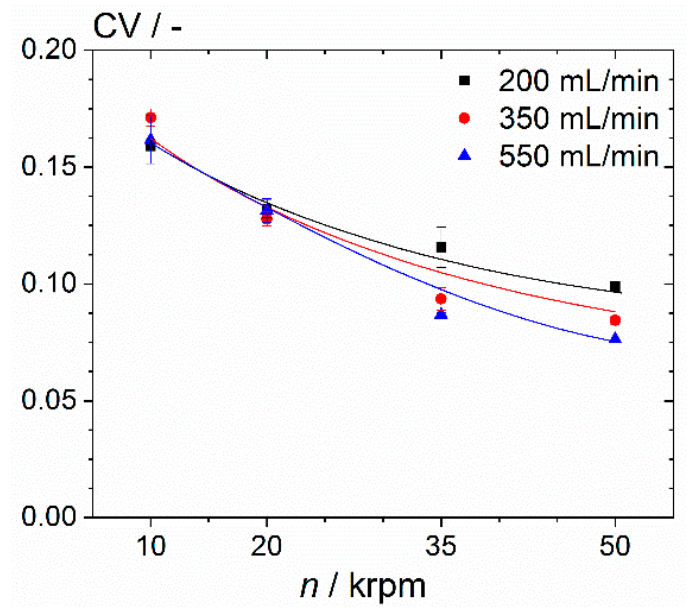

Figure 7. Coefficient of variation calculated from time-dependent spray area vs. nominal rotational speed $\mathrm{n}$ for different volumetric flow rates $\dot{V}$.

Figure 8 demonstrates that the arithmetic mean droplet diameter $D_{10}$ of the sprayed basecoat decreases with increasing rotational speed as already observed in many studies on rotary atomization [1,2,14-18]. Elongation of ligaments is enhanced with increasing rotational speed. As a result, ligaments become thinner before final breakup, i.e., droplet diameter decreases [19]. On the other hand, volumetric flow rate does not affect droplet size significantly. Increasing volumetric flow rate increases initial ligament diameter $d_{1}$, however, capillary breakup time $t_{\text {cap }}$ increases as well according to $t_{\text {cap }}=\left(\rho d_{1}^{3} / \sigma\right)^{1 / 2}$ [20]. As a result of these contrary effects, the dependence of droplet size on volumetric flow rate is weak.

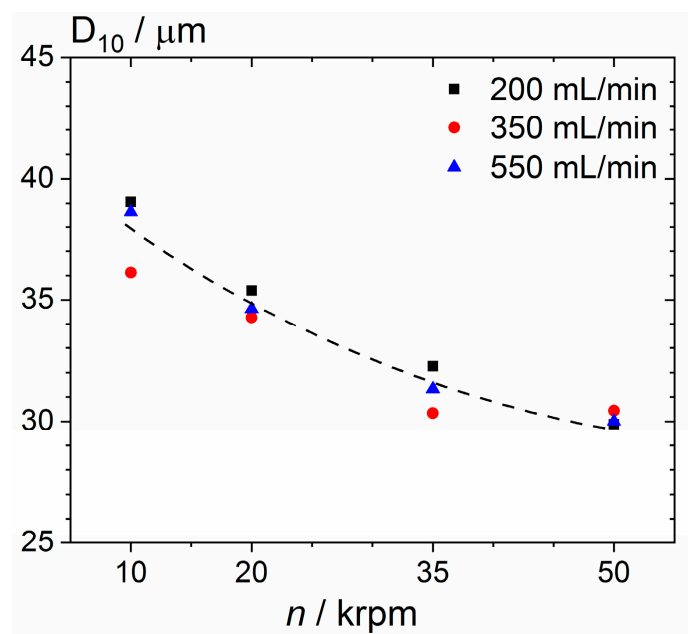

Figure 8. Arithmetic mean droplet diameter $D_{10}$ of the waterborne basecoat as a function of rotational speed $n$ for different volumetric flow rates $\dot{V}$. 
The arithmetic mean droplet diameter was not affected strongly by paint flow pulsations. The fraction of large droplets, however, may be related to the degree of fluctuation. Generally, the fraction of large droplets increases with decreasing nominal rotational speed since not only $D_{10}$ but the whole droplet size distribution shifted to higher values. However, Figure 9 shows that the fraction of droplets with diameter larger than $100 \mu \mathrm{m}$ increases almost linearly with increasing coefficient of variation. Hence, we conclude that the formation of large droplets is promoted by fluctuations of the flowing liquid and not only by rotational speed.

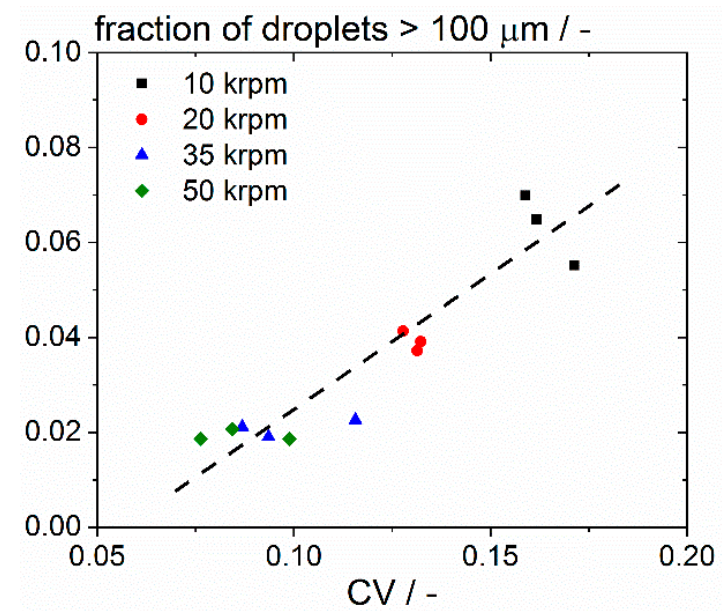

Figure 9. Fraction of droplets larger than $100 \mu \mathrm{m}$ vs. coefficient of variation CV for different rotational speeds $n$. The line is to guide the eye.

This section may be divided into subheadings. This should provide a concise and precise description of the experimental results, their interpretation, as well as the experimental conclusions that can be drawn.

\section{Summary and Conclusions}

High-speed bell atomization is the global standard in the automotive industry for the coating of car bodies. In this process, a uniform coating layer is ensured by a homogenous distribution of the spray on the substrate. However, this can be impaired by the presence of paint flow fluctuations. To shed some light on this phenomenon, we have investigated pulsations during atomization of an automotive coating based on high-speed imaging of the spray.

Therefore, 3000 images were recorded with a frame rate of 100,000 fps near the bell edge and subsequently processed and analyzed by a customized MATLAB code. A Fourier transformation was applied on the time evolution of white pixel area during atomization in order to obtain the corresponding frequency spectrum (Figure 4). Additionally, the coefficient of variation of the spray was determined in order to quantify the degree of fluctuation.

We have shown that the main frequency increases linearly with increasing rotational speed. Normalizing these data with the true rotational speed itself resulted in a value of approximately 3 . This indicates that the three struts connecting the distributor disc with the bell cup are the source of the observed pulsations. The degree of fluctuation is also affected by the application parameters. Increasing rotational speed and volumetric flow rate decreased the coefficient of variation of the spray area in the recorded images. We suggest that increasing centrifugal force promotes the levelling of the film at the bell edge, and therefore reduces relative amplitudes of pulsations. Finally, we determined that the formation of droplets with d larger than $100 \mu \mathrm{m}$, which are assumed to cause paint defects, is promoted by the degree of fluctuation. These findings can serve as a basis for the development of bell cups showing less paint flow pulsations. 
Author Contributions: Conceptualization, W.O. and N.W.; Methodology, W.O., J.L., L.G., P.E. and N.W.; Software, J.L. and W.O.; Validation, J.L., W.O. and N.W.; Investigation, W.O., J.L. and L.G.; Writing-Original Draft Preparation, W.O. and N.W.; Writing-Review and Editing, W.O. and N.W.; Visualization, W.O.; Supervision, N.W.

Funding: This research was funded by BASF Coatings $\mathrm{GmbH}$ and we acknowledge support by the KIT-Publication Fund of the Karlsruhe Institute of Technology.

Acknowledgments: The authors would like to thank BASF Coatings $\mathrm{GmbH}$ for access to their spraying facilities including high-speed imaging and droplet size determination as well as financial support. Fruitful discussions with Georg Wigger and Daniel Briesenick (BASF Coatings $\mathrm{GmbH}$ ) are gratefully acknowledged.

Conflicts of Interest: The funding company, BASF coatings GmbH did not take any influence on the design of the study; in the collection, analysis, or interpretation of data.

\section{References}

1. Milborn, H.; Hinze, J. Atomization of liquids by means of a rotating cup. J. Appl. Mech. Tran. ASME 1950, 17, 145-153.

2. Frost, A.R. Rotary atomization in the ligament formation mode. J. Agric. Eng. Res. 1981, 26, 63-78. [CrossRef]

3. Ogasawara, S.; Daikoku, M.; Shirota, M.; Inamura, T.; Saito, Y.; Yasumara, K.; Shoji, M.; Aoki, H.; Miura, T. Liquid atomization using a rotary bell cup atomizer. J. Fluid Sci. Technol. 2010, 5, 464-474. [CrossRef]

4. Kourmatzis, A.; Lowe, A.; Masri, A.R. Combined effervescent and airblast atomization of a liquid jet. Exp. Therm. Fluid Sci. 2016, 75, 66-76. [CrossRef]

5. Ariyapadi, S.; Balachandar, R.; Berruti, F. Characterizing spray pulsations using a phase-doppler particle analyzer. Ind. Eng. Chem. Res. 2001, 40, 5282-5290. [CrossRef]

6. Schlinge, B.; Schröder, J.; Gaukel, V.; Schuchmann, H.P.; Walzel, P. Vergleich von Methoden zur Pulsationsmessung an innenmischenden Zweistoffdüsen. Chemie Ing. Tech. 2013, 10, 1568-1574. [CrossRef]

7. Schröder, J.; Günther, A.; Wirth, K.-E.; Schuchmann, H.P.; Gaukel, V. Effervescent atomization of polyvinylpyrrolidone solutions: Influence of liquid properties and atomizer geometry on liquid breakup and spray characteristics. At. Sprays 2013, 23, 1-23. [CrossRef]

8. Mullis, A.M.; Cochrane, R.F.; McCarthy, I.N.; Adkins, N.J. Log-normal melt pulsation in close-coupled gas atomization. Metall. Mater. Trans. B Process Metall. Mater. Process. Sci. 2013, 44, 789-793. [CrossRef]

9. Ting, J.; Connor, J.; Ridder, S. High-speed cinematography of gas-metal atomization. Mater. Sci. Eng. A 2005, 390, 452-460. [CrossRef]

10. Im, J.-H.; Kim, D.; Han, P.; Yoon, Y.; Bazarov, V. Self-pulsation characteristics of a gas-liquid swirl coaxial injector. At. Sprays 2009, 19, 57-74. [CrossRef]

11. Kang, Z.; Li, Q.; Cheng, P.; Zhang, X.; Wang, Z. Effects of self-pulsation on the spray characteristics of gas-liquid swirl coaxial injector. Acta Astronaut. 2016, 127, 249-259. [CrossRef]

12. Soma, T.; Katayama, T.; Tanimoto, J.; Saito, Y.; Matsushita, Y.; Aoki, H.; Nakai, D.; Kitamura, G.; Miura, M.; Asakawa, T.; et al. Liquid film flow on a high speed rotary bell-cup atomizer. Int. J. Multiph. Flow 2015, 70, 96-103. [CrossRef]

13. Salazar, A. Computational modeling of relevant automotive rotary spray painting process. In Automotive Painting Technology: A Monozukuri-Hitozukuri Perspective, 1st ed.; Toda, K., Salazar, A., Saito, K., Eds.; Springer: Dordrecht, Netherlands, 2013; pp. 47-95.

14. Oswald, W.; Gödeke, L.; Ehrhard, P.; Willenbacher, N. An experimental study of the influence of elongational flow resistance and pigmentation of coating fluids on high-speed rotary bell atomization. At. Sprays 2019. submitted.

15. Liu, Y.; Lai, M.-C.; Im, K.-S.; Sankagiri, N.; Loch, T.; Nivi, H. An Experimental Investigation of Spray Transfer Processes in an Electrostatic Rotating Bell Applicator; SAE International: Warrendale, PA, USA, September 1998.

16. Ellwood, K.R.J.; Tardiff, J.L.; Alaie, S.M.A. Simplified analysis method for correlating rotary atomizer performance on droplet size and coating appearance. J. Coatings Technol. Res. 2014, 11, 303-309. [CrossRef]

17. Ryley, D.J. Analysis of a polydisperse aqueous spray from a high-speed spinning disk atomizer. Br. J. Appl. Phys. 1959, 10, 180-186. [CrossRef]

18. Ahmed, M.; Youssef, M.S. Characteristics of mean droplet size produced by spinning disk atomizers. J. Fluids Eng. 2012, 134, 071103. [CrossRef] 
19. Gramlich, S.; Piesche, M. Numerical and experimental investigations on the breakup of particle laden liquid jets in the centrifugal field. Chem. Eng. Sci. 2012, 84, 408-416. [CrossRef]

20. Kuhnhenn, M.; Joensen, T.V.; Reck, M.; Roisman, I.V.; Tropea, C. Study of the internal flow in a rotary atomizer and its influence on the properties of the resulting spray. Int. J. Multiph. Flow 2018, 100, 30-40. [CrossRef] 